\title{
Adaptação Paramétrica Evolucionária no Controle Adaptativo Livre de Modelo *
}

\author{
Elaine Guerrero-Peña* Aluizio F.R. Araújo** \\ * Centro de Informática, Universidade Federal de Pernambuco, PE, \\ (e-mail: egp@cin.ufpe.br). \\ ** Centro de Informática, Universidade Federal de Pernambuco, PE, \\ (e-mail: aluizioa@cin.ufpe.br).
}

\begin{abstract}
This paper considers the use of Model-Free Adaptive Control (MFAC) for the Continuously Stirred Tank Reactor, a nonlinear system working with and without disturbances. Finding the optimal set of MFAC parameters is still a complex open problem that may be subject to multiple conflicting requirements. However, only a small number of approaches proposed in the literature consider this adjustment task as a multi-objective problem. Using a Multi-objective Evolutionary Algorithm (MOEA) seems to be an appropriate approach to adjust the MFAC. However, MOEA convergence time is an essential feature to make it viable for application in the systems control. Therefore, in this paper, we use the Gaussian Process Regression-based Evolutionary Algorithm (GPR-EA). GPR-EA considers past generations to establish trends in population movement and thus find the approximate Pareto front more quickly. Simulations illustrate the effectiveness and efficiency of the MFAC with the tuning performed with GPR-EA.

Resumo: Este artigo considera o emprego do Controle Adaptativo Livre de Modelo (MFAC) para o Reator Tanque Agitado Contínuo, um sistema não-linear atuando com e sem distúrbios. Encontrar o conjunto ótimo de parâmetros do MFAC é um problema complexo em aberto, que pode estar sujeito a múltiplos requisitos conflitantes. Contudo, apenas um pequeno número das abordagens propostas na literatura consideram esta tarefa de ajuste como um problema multiobjetivo. Usar um Algoritmo Evolucionário Multiobjetivo (MOEA) parece ser uma abordagem adequada para ajustar o MFAC. No entanto, o tempo de convergência do MOEA é um elemento essencial para seu emprego em controle de sistemas. Por esse motivo, neste trabalho usamos o MOEA Gaussian Process Regression-based Evolutionary Algorithm (GPR-EA). GPR-EA considera as gerações passadas para estabelecer tendências no movimento populacional e, assim, encontrar mais rapidamente a frente de Pareto aproximada. A eficácia e eficiência do controle MFAC com o ajuste realizado com o GPR-EA é ilustrada por simulações.
\end{abstract}

Keywords: Model Free Adaptive Control; Intelligent Control; Parameter Optimization; Multi-Objective Evolutionary Optimization; Gaussian Process Regression.

Palavras-chaves: Controle Adaptativo Livre de Modelo; Controle Inteligente; Otimização de Parâmetros; Otimização Multiobjetivo Evolucionária; Regressão do Processo Gaussiano.

\section{INTRODUÇÃO}

Com o desenvolvimento da informática e da tecnologia, muitos processos industriais tornaram-se cada mais complexos, e os requisitos sobre a qualidade dos produtos passaram a ser mais demandantes. Modelar esses processos usando os métodos de identificação tradicional torna-se cada vez mais difícil, até mesmo impraticável porque o modelo pode ter uma ordem muito alta, alta não-linearidade e conter estruturas mais complexas (Hou and Jin, 2013). Quando modelos precisos do processo são muito difíceis de construir ou não estão disponíveis, métodos de Data Driven Control (DDC) são de importância significativa tanto na teoria de controle quanto nas aplicações. DDC foi definido em Hou and Jin (2013) como "a teoria e método de controle, no qual o controlador é projetado diretamente usando dados de Entrada/Saída online ou offline do sis-

\footnotetext{
* Este trabalho foi apoiado por uma bolsa de estudos da CAPES.
}

tema controlado, ou o conhecimento do processamento de dados sem usar explicitamente as informações do modelo matemático do processo controlado e cuja estabilidade, convergência e robustez podem ser garantidas por uma análise rigorosa sob certas suposições razoáveis".

Dentre tais abordagens DDC, o MFAC é um controle com uma estrutura sistemática e rigorosa, efetivo para sistemas não-lineares de tempo discreto com crescente interesse nos últimos anos (Hou and Jin, 2013). Neste tipo de controle, o projeto do controlador não demanda um modelo explícito do sistema a ser controlado. O sistema MFAC requer que o processo seja controlável, estável em malha aberta e seja de atuação direta ou reversa (Uçak and Günel, 2019). Não obstante o MFAC ser promissor (Uçak and Günel, 2019; de Sousa et al., 2015), seu projeto é complexo por se basear apenas nos dados de entrada e saída para sistemas desconhecidos. Encontrar o conjunto ótimo de parâmetros do MFAC é um problema complexo em aberto. 
Em geral, esses parâmetros são selecionados com base em uma análise qualitativa da resposta do sistema. O ajuste dos parâmetros de um MFAC melhorado sem considerar o ruído foi discutido preliminarmente em Wang et al. (2012), mas ele só é direcionado ao modelo universal de segunda ordem. Considerando o ruído aleatório existente em processos reais, um método de ajuste de parâmetro baseado na otimização de entropia mínima é proposto em Ji et al. (2014). Em Roman et al. (2016), um método de ajuste de parâmetros off-line foi proposto usando um algoritmo não linear de sintonia de realimentação de referência virtual (VRFT). Em Chen et al. (2019) é proposta uma nova abordagem de auto-ajuste de parâmetros do MFAC baseada numa rede neural de propagação reversa com erro de sistema configurado como entrada.

Outras estratégias para encontrar os parâmetros ótimos do MFAC incluem emprego de Algoritmos Evolucionários (EAs) (dos Santos Coelho and Coelho, 2009; de Sousa et al., 2015). A configuração dos parâmetros MFAC pode estar sujeita a requisitos conflitantes. Por exemplo, um conjunto de parâmetros pode reduzir simultaneamente o erro de estado estacionário e aumentar o erro máximo em períodos transitórios. Portanto, um MOEA pode encontrar soluções de compromisso. No entanto, a maioria das abordagens propostas consideram um problema de objetivo único e apenas um pequeno número de artigos consideram o problema multiobjetivo (de Sousa et al., 2015). A otimização multiobjetivo permite escolher diferentes soluções de compromisso para os parâmetros causando diferentes desempenhos do sistema. Assim, o engenheiro de controle pode escolher o melhor conjunto de parâmetros dependendo dos requerimentos do controle.

Neste trabalho, foi considerado o problema de ajuste dos parâmetros do MFAC para um sistema não-linear sujeito a perturbações: o Reator Tanque Agitado Contínuo. Aqui a ocorrência de perturbações pode não ser acuradamente capturada nos modelos. Assim, é muito desejável que o controlador MFAC projetado seja insensível a essas incertezas. Para ajustar os parâmetros do MFAC, empregase o Gaussian Process Regression-based Evolutionary Algorithm (GPR-EA) desenvolvido em Guerrero-Peña and Araújo (2018). GPR-EA determina as tendências de movimentação populacional em gerações anteriores para buscar soluções próximas àquelas ótimas de Pareto. Os resultados das simulações sugerem a eficácia e eficiência do GPR-EA para determinar os parâmetros ótimos do controlador que foi testado para o rastreamento para diferentes sinais de referência na presença de distúrbios.

O restante deste artigo está organizado da seguinte forma. A Seção 2 descreve os princípios básicos do MFAC. A Seção 3 apresenta o GPR-EA empregado para ajustar os parâmetros do MFAC. O modelo do caso de estudo e a configuração do GPR-EA são mostrados na Seção 4. A Seção 5 apresenta os resultados da simulação, a análise do desempenho do controlador MFAC e a comparação com outros controladores da literatura. O documento termina com uma breve conclusão na Seção 6 .

\section{CONTROLE ADAPTATIVO LIVRE DE MODELO}

O controle adaptativo livre de modelo (Model-Free Adaptive Control: MFAC) apenas os dados de entrada e saída da planta controlada são diretamente usados para projetar um controlador e para a análise do sistema em malha fechada, sem qualquer dinâmica de modelo envolvida.

Considere o seguinte sistema não-linear de uma entrada e uma saída (SISO) de tempo discreto (Hou and Jin, 2013):

$$
y(k+1)=f\left(y(k), \cdots, y\left(k-n_{y}\right), u(k), \cdots, u\left(k-n_{u}\right)\right)
$$

onde $n_{y}, n_{u}$ são as ordens desconhecidas da saída $y(k)$ e da entrada $u(k)$ do sistema no instante $k$, respectivamente, e $f(\cdot)$ é uma função não-linear desconhecida.

As seguintes suposições são feitas para a planta controlada (Hou and Jin, 2013): S1: a derivada parcial de $f(\cdot)$ em relação à entrada do controle $u(k)$ é contínua. $\mathrm{S} 2$ : o sistema 1 é generalizado Lipschitz, isto é, $|\Delta y(k+1)| \leq b|\Delta u(k)|$ para qualquer $k$ e $\Delta u(k) \neq 0$, com $\Delta y(k+1)=y(k+1)-$ $y(k), \Delta u(k)=u(k)-u(k-1)$. A constante $b$ é positiva.

Um sistema discreto não-linear que satisfaça as suposições S1-S2 pode ser transformado em um modelo de linearização dinâmica de forma compacta (Compact Form Dynamic Linearization. Este CFDL constrói uma série de modelos lineares dinâmicos e equivalentes ao longo dos pontos dinâmicos de operação da planta controlada, ao invés de identificar um único modelo global não-linear do processo. Assim, se um sistema não-linear da Eq. 1 satisfizer as S1 e S2, então deve existir uma pseudo-derivada parcial (Pseudo-Partial-Derivative - PPD) $\phi(k)$, tal que se $\Delta u(k) \neq 0$, o sistema da Eq. 1 pode ser descrito pelo modelo CFDL (Hou and Jin, 2013):

$$
y(k+1)=y(k)+\phi(k) \Delta u(k) \operatorname{com}|\phi(k)| \leq b
$$

A Eq. 2 é um sistema linear dinâmico com um parâmetro de variação temporal lenta, se a taxa de mudança do sinal de entrada $\Delta u(k) \neq 0$ e não for muito alta.

Uma função objetivo é usada para a estimação dos parâmetros da $\operatorname{PPD} \phi(k)$ :

$J(\phi(k))=|y(k)-y(k-1)-\phi(k) \Delta u(k-1)|^{2}+\mu|\phi(k)-\hat{\phi}(k-1)|^{2}$

onde $\mu$ é um fator de peso positivo. Assim, para qualquer instante $k$, pode-se estimar a PPD (usando apenas os dados de I/O da planta controlada) como:

$$
\hat{\phi}(k)=\hat{\phi}(k-1))+\frac{\eta \Delta u(k-1)}{\mu+|\Delta u(k-1)|^{2}}(\Delta y(k)-\hat{\phi}(k-1) \Delta u(k-1))
$$

onde $\eta \in(0,1)$ é o tamanho do passo.

Para que a condição $\Delta u(k) \neq 0$ seja satisfeita e, consequentemente fazer com que o algoritmo de estimação de parâmetros consiga rastrear o parâmetro variante no tempo, a seguinte condição de reinicialização deve ser considerada (Hou and Jin, 2013):

$$
\begin{gathered}
\hat{\phi}(k)=\hat{\phi}(\mathbf{1}), \text { se }|\hat{\phi}(k)| \leq \epsilon \text {, ou }|\Delta u(k-1)| \leq \epsilon, \\
\text { ou } \operatorname{sign}(\hat{\phi}(k)) \neq \operatorname{sign}(\hat{\phi}(\mathbf{1}))
\end{gathered}
$$

onde $\hat{\phi}(\mathbf{1})$ é o valor inicial para o PPD estimado e $\epsilon$ é uma constante pequena positiva.

Para o esquema de controle, uma função ponderada de custo de entrada de um passo é adotada e dada por:

$$
J(u(k))=\left|y^{*}(k+1)-y(k+1)\right|^{2}+\lambda|u(k)-u(k-1)|^{2}
$$

onde $y^{*}(k+1)$ é o sinal de referência ou sinal esperado da saída do sistema, e $\lambda$ é um fator de peso positivo. 
A substituição de (2) em (6) e a resolução da equação $\frac{\partial J(u(k))}{\partial u(k)}=0$, fornece o sinal de controle (Hou and Jin, 2013):

$$
u(k)=u(k-1)+\frac{\rho \phi(k)}{\lambda+|\phi(k)|^{2}}\left(y^{*}(k+1)-y(k+1)\right)
$$

onde $\rho \in(0,1)$ é o tamanho do passo.

\section{ALGORITMO EVOLUCIONÁRIO BASEADO NA REGRESSÃO POR PROCESSO GAUSSIANO}

Ao resolver um problema de otimização multiobjetivo um Algoritmo Evolucionário (EAs) tende a perder a diversidade com o avanço do processo evolucionário, levando o algoritmo à convergência prematura. Portanto, equilibrar os níveis de exploração e a explotação é necessário como ocorre no Algoritmo Evolucionário Baseado na Regressão por Processo Gaussiano (do inglês Gaussian Process Regression-based Evolutionary Algorithm: GPR-EA) desenvolvido em Guerrero-Peña and Araújo (2018).

Inicialmente, os parâmetros do GPR-EA são definidos e, a população inicial é gerada, avaliada e ordenada usando o conceito de dominância de Pareto. Enquanto o critério de parada não for satisfeito, todo o processo evolucionário é repetido. Um critério baseado na métrica de Hypervolume (HV) (While et al., 2006) é usado para selecionar o método de geração de soluções candidatas. Esta métrica é calculada aproximadamente em cada geração com as soluções não-dominadas. O critério se baseia na taxa de crescimento do HV com um limiar adaptativo e com um intervalo de gerações variáveis para a regressão. O procedimento GPR é acionado se:

$$
\text { GPR se }\left\{\begin{array}{l}
H V(g) \geq \text { Limiar } \\
\&\left(g-\text { ger }_{\text {init }}\right) \geq 3
\end{array}\right.
$$

com: Limiar $=H V\left(\right.$ ger $\left._{\text {init }}\right)+$ perc $_{\text {var }} * H V\left(\right.$ ger $\left._{\text {init }}\right) \quad(9)$ onde $g$ é a geração atual, ger ${ }_{i n i t}$ é a geração de referência; $\operatorname{perc}_{\text {var }}$ representa a percentagem de aumento do $\mathrm{HV}(\mathrm{g})$ em relação ao $H V\left(g e r_{i n i t}\right)$. A condição $\left(g-g e r_{i n i t}\right) \geq 3$ garante que pelo menos três gerações são usadas para estimar a direção para o processo evolucionário com GPR. Para cada geração, o ponto de referência para calcular HV é escolhido como o valor máximo de todos os objetivos. Embora o valor de HV seja sensível a esse ponto de referência, deve-se enfatizar que a determinação do HV não necessita ser acurada, pois o desejado é ter uma avaliação aproximada da convergência do algoritmo.

Se este critério de regressão for satisfeito, executa-se o processo de regressão no espaço de decisão usando um Processo Gaussiano (GP) e a distribuição preditiva. Assim, em determinadas gerações, obtém-se um conjunto de novos vetores é obtido considerando-se informações das gerações passadas do processo evolucionário. O primeiro passo é estimar o modelo GP usando as soluções de populações consecutivas $P\left(\right.$ ger $_{\text {init }}:$ ger $\left._{\text {end }}\right)$ :

$$
p(\theta \mid x, \alpha) \sim \mathcal{G P}\left(m(x), k\left(x, x^{\prime}\right)\right)
$$

onde $\theta=\left[\bar{m}^{*}, k\left(x^{*}, x^{*}\right)\right]$ é o vetor de parâmetros do modelo e $\alpha$ é o vetor de hiperparâmetros. A distribuição de um GP é especificada por uma função média $m(x)$ e uma função de covariância $k\left(x, x^{\prime}\right)$ (Rasmussen and Williams, 2006).
Então, novos vetores são gerados mediante a Regressão de Processo Gaussiano usando a distribuição preditiva:

$$
p\left(x^{*} \mid x\right)=\int_{\theta} p\left(x^{*} \mid \theta, x\right) p(\theta \mid x, \alpha) d \theta
$$

Por fim, as soluções não viáveis são reparadas, criando o conjunto de soluções candidatas $Q_{t}$ que acelera a convergência do algoritmo podendo levar a soluções aproximadas àquelas ótimas de Pareto mais rapidamente.

Após a execução da regressão, os parâmetros do critério de regressão são atualizados da seguinte forma:

- Nova geração inicial para a regressão: ger $_{\text {init }}=g$,

- Limite ajustado de acordo com a Equação 9,

- $\operatorname{perc}_{v a r}=\operatorname{perc}_{v a r}+0,025$.

Se o critério de regressão não for acionado, as soluções candidatas são geradas usando a mutação diferencial e recombinação binomial. Na próxima etapa, o operador de mutação polinomial é aplicado. Os operadores do DE têm predominância exploratória enquanto a mutação polinomial tem atuação explotativa (Guerrero-Peña and Araújo, 2018). Os conjuntos de soluções atuais $P_{t}$ e de novas soluções $Q_{t}$ são combinadas para formar um conjunto de tamanho $2 N$. Este conjunto é ordenado e as soluções são classificadas por não-dominância em diferentes frentes $F_{k}$. Após a conclusão da ordenação, a nova população $P_{t+1}$, de tamanho $N$, é gerada a partir das soluções no conjunto não-dominado. Para comparar soluções com a mesma classificação e ocupantes das últimas posições em $P_{t+1}$, usamos um estimador de densidade baseado no método $K$-means (Lloyd, 1982)). Assim, as soluções mais diferentes são selecionadas para as últimas posições disponíveis de $P_{t+1}$ mantendo a diversidade da população.

\section{CONFIGURAÇÃO DA SIMULAÇÃO}

Nesta seção são descritos o caso de estudo não-linear com perturbações $(d(t))$ e os sinais de referências usados para testar a eficácia e a eficiência do GPR-EA na determinação dos parâmetros do controlador MFAC. A perturbação $d(t)$ é simulada por um ruído Gaussiano de média zero com 30dB SNR adicionado à saída controlada de cada sistema. Também são descritas a representação da solução usada pelo GPR-EA e as funções de aptidão empregadas para a avaliação de soluções candidatas. Finalmente, é apresentado o tomador de decisão usado para escolher o conjunto de parâmetros ótimos do MFAC, entre as soluções da fronteira de Pareto aproximadas pelo GPR-EA.

\subsection{Caso de Estudo: Reator Tanque Agitado Contínuo.}

O Sistema de Reator Tanque Agitado Contínuo (CSTR) é um tipo de reator químico no qual são realizadas reações químicas sucessivas multicomponentes, isotérmicas e em fase líquida. Os conteúdos no reator são agitados e uniformes em todo o processo. Este tipo de sistema é muito utilizado na indústria para produzir polímeros, produtos farmacêuticos e outros produtos químicos. Os reagentes de entrada (A, B) são misturados em um recipiente com volume constante através de um agitador e retornam o produto C. Nesta planta, o objetivo é controlar a concentração do produto $\mathrm{C}$ ajustando a taxa molar de alimentação do 
reagente $\mathrm{B}$. O comportamento dinâmico do CSTR nãolinear de terceira ordem pode ser expresso através das equações diferenciais (Uçak and Günel, 2019):

$$
\begin{gathered}
\dot{x}_{1}=1-4 x_{1}+0,5 x_{2}^{2} \\
\dot{x}_{2}=-x_{2}+3 x_{1}-1,5 x_{2}^{2}+u(t) \\
\dot{x}_{3}=-x_{3}+x_{2}^{2}+d(t)
\end{gathered}
$$

onde $x_{1}(t), x_{2}(t)$, e $x_{3}(t)$ denotam os estados obtidos das concentrações dos reagentes $\mathrm{A}$ e $\mathrm{B}$ e do produto C. $u(t)$ é o sinal de controle, e $x_{3}(t)$ é a saída do sistema.

\subsection{Sinais de Referência}

O controlador foi projetado para o rastreamento dos seguintes sinais de referência:

(1) Sinal de escada

$$
y^{*}(k+1)= \begin{cases}0,4, & k \leq 100 \text { e } 400<k \leq 500 \\ 0,5, & 100<k \leq 200 \text { e } 300<k \leq 400 \\ 0.6, & 200<k \leq 300\end{cases}
$$

(2) Sinal senoidal

$$
\begin{aligned}
y^{*}(k+1)= & 0.15 \cdot \operatorname{sen}(2 * \pi / f(k))+0.5 \\
f(k)= & \begin{array}{ll}
310, & 0<k \leq 310 \\
190, & 310<k \leq 500 \\
100, & 500<k \leq 600 \\
80, & 600<k \leq 680 \\
30, & 680<k \leq 710 \\
40, & 710<k \leq 750 \\
30, & 750<k \leq 780 \\
20, & 780<k \leq 800
\end{array}
\end{aligned}
$$

\subsection{Configuração do GPR-EA}

Uma solução do problema é representada por um vetor $x \in R^{4}$ contendo os parâmetros reais das equações (4) e $(7): \rho \in(0,1], \eta \in(0,1], \mu \in(0,10], \lambda \in(0,10]$. O GPR-EA foi executado 10 vezes independentes durante 50 gerações. A população é formada por 20 soluções. No GPREA deste artigo, os demais parâmetros são a probabilidade de mutação de $p_{m}=1 / D$; índice de distribuição $\eta_{m}=20$ para o operador de mutação polinomial; além de tamanho de passo de mutação $F=0,5$ e taxa de cruzamento $C R=$ 1 nos operadores da evolução diferencial. A percentagem de variação $\operatorname{perc}_{\text {var }}$ foi definida como 0,1 .

\subsection{Funções Objetivo}

No trabalho foram usadas duas funções objetivos propostas em de Sousa et al. (2015). A primeira (Eq. 13), definida como a média do sinal de erro acumulado ao quadrado, é usada para minimizar o erro de estado estacionário. A segunda função (Eq. 14) busca reduzir o máximo erro durante o estado de transição. Esta função é calculada usando a diferença quadrática acumulada entre as derivadas dos sinais de referência e de saída.

$$
\begin{gathered}
f_{1}\left(y^{*}(k), y(k)\right)=\frac{1}{N} \sum_{k=1}^{N}\left[e(k)^{2}\right], \text { com } e(k)=y^{*}(k)-y(k) \\
f_{2}\left(y^{* \prime}(k), y^{\prime}(k)\right)=\frac{1}{N-1} \sum_{k=1}^{N-1}\left[\left(y^{* \prime}(k)-y^{\prime}(k)\right)^{2}\right]
\end{gathered}
$$

\subsection{Tomador de Decisão}

O GPR-EA produz uma frente de Pareto aproximada $(\mathrm{PF})$, portanto, uma única solução deve ser selecionada entre as várias opções do conjunto de Pareto. Frequentemente, o ponto de joelho (knee-point) na PF é a solução escolhida se o tomador de decisão não tiver uma preferência especial. Portanto, empregamos um método de knee-point (Distance to the extreme line method), que seleciona a solução $\left(x^{*}\right)$ mais próxima de um dado ponto de referência, em geral, a origem de coordenadas (Liang et al., 2018):

$$
\left.x^{*}=\min _{1 \leq i \leq N P}\left(d\left(x_{i}\right)\right) \mid d_{i}=\sqrt{(} f_{1_{N}}\left(x_{i}\right)^{2}+f_{2_{N}}\left(x_{i}\right)^{2}\right)
$$

onde $f_{1_{N}}$ e $f_{2_{N}}$ são as funções objetivo normalizadas.

\section{RESULTADOS DA SIMULAÇÃO}

Nesta secção, são apresentadas as soluções obtidas pelo algoritmo evolucionário e a resposta do sistema controlado usando os parâmetros escolhidos. Além disso, há comparações com métodos de controle livre de modelo e baseado em modelo.O método ode23tb ${ }^{1}$ do MatLab foi empregado nas simulações com o período de amostragem de 0,1s. As condições iniciais do Reator Tanque Agitado Contínuo controlado são: $x_{1}=0,8796 ; x_{2}=0,8796 ; x_{3}=$ 0,$3467 ; u_{1}=0 ; \phi(\mathbf{1})=1 ;$ e $\epsilon=10^{-5}$.

Os valores das funções de aptidão e do erro absoluto máximo foram analisados (Tabela 1) para diferentes soluções entre as várias opções do conjunto de Pareto aproximadas pelo GPR-EA. Todos os conjuntos de parâmetros escolhidos permitem ao sistema não-linear seguir o sinal de referência. A solução escolhida pelo tomador de decisão $\left(s_{T D}\right)$ é a mais próxima do ponto de utopia. Este conjunto de parâmetros possibilita que o MFAC apresente o melhor compromisso encontrado entre o erro estacionário $\left(f_{1}\right)$ e o sobressinal $\left(f_{2}\right)$. A solução que minimiza a primeira função de aptidão $\left(s_{f_{1}}\right)$, reduz o erro de estado estacionário, com o custo de aumentar o período transitório; enquanto que a solução que minimiza o segundo índice $\left(s_{f_{2}}\right)$, tem o efeito oposto. No rastreamento de sinais sem ruído, o máximo valor do erro absoluto é similar para as diferentes soluções. Este erro é encontrado nos primeiros instantes de tempo. No entanto, para o caso do sinal com ruído, o valor máximo do erro absoluto e o tempo em que este ocorre, varia com a solução escolhida. Neste caso, o desempenho do controlador é mais sensível à escolha da solução. Assim, a melhor seleção paramétrica depende dos requisitos do controle.

Para cada sinal de referência, as soluções escolhidas pelo tomador de decisão dentre as produzidas pelo GPR-EA estão na Tabela 2. O desempenho de rastreamento do controlador MFAC para os sinais de referência, quando nenhum ruído é aplicado, e os sinais de controle em resposta a estes sinais de referência estão na Figura 1. O controlador rastreou com precisão no caso sem ruído. A robustez do sistema considerando o ruído Gaussiano branco com SNR $=30 \mathrm{Db}$ está ilustrada na Figura 2 para sinais de referência escada e senoidal: a dinâmica do sistema rastreia o sinal de referência; apesar da flutuação, o sinal de saída converge.

1 Resolve equações diferenciais rígidas usando regra trapezoidal e a fórmula de diferenciação inversa 

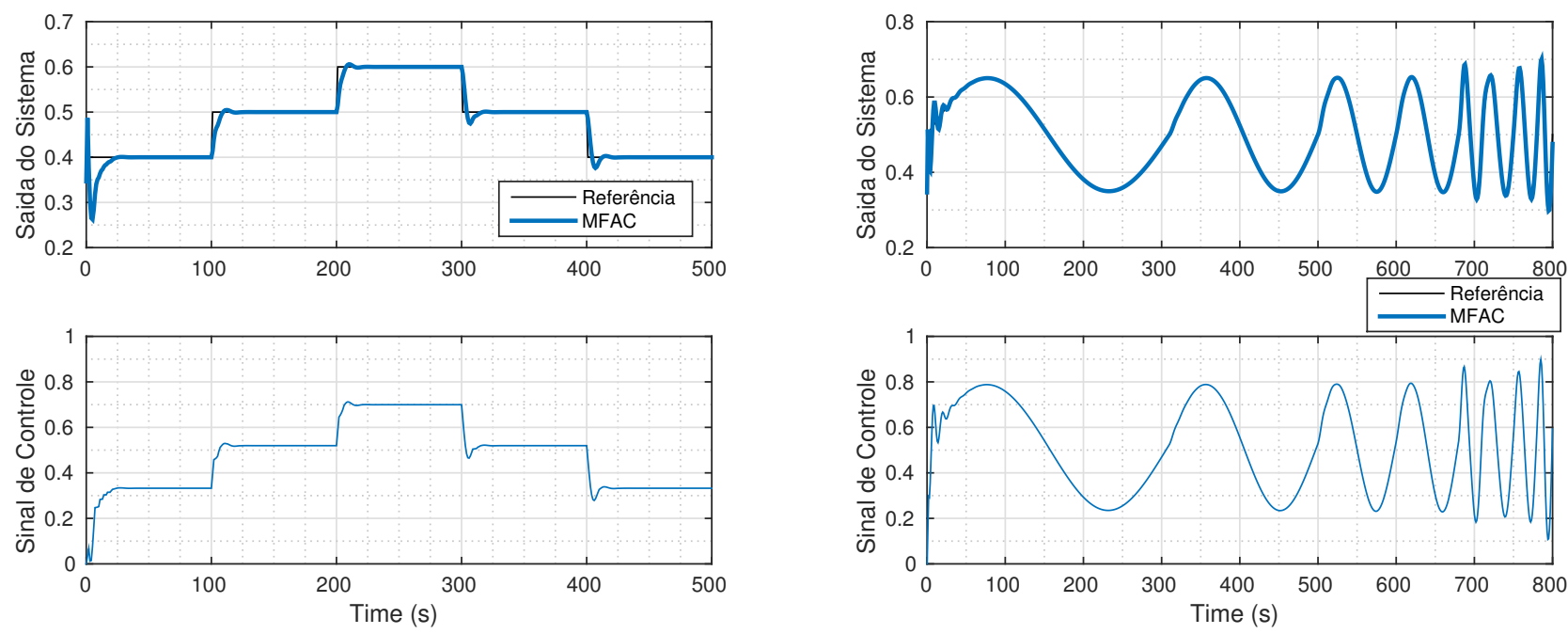

(a) Referência Escada

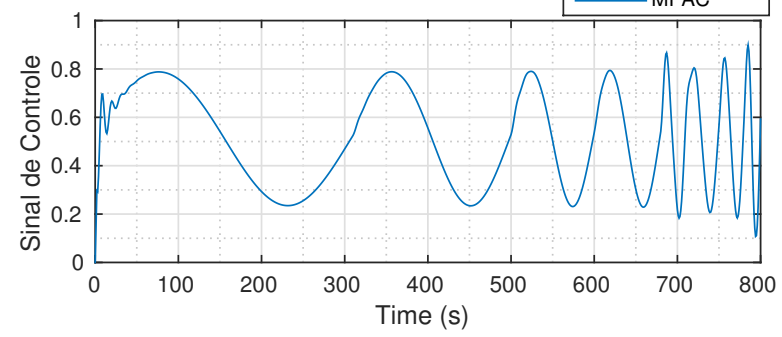

(b) Referência Senoidal

Figura 1. Rastreamento do controlador MFAC sem ruído, para os sinais de referência e sinais de controle.
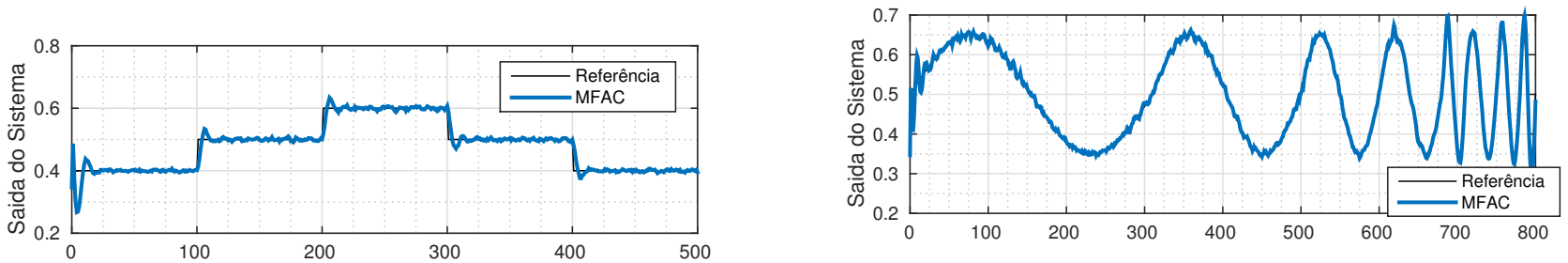

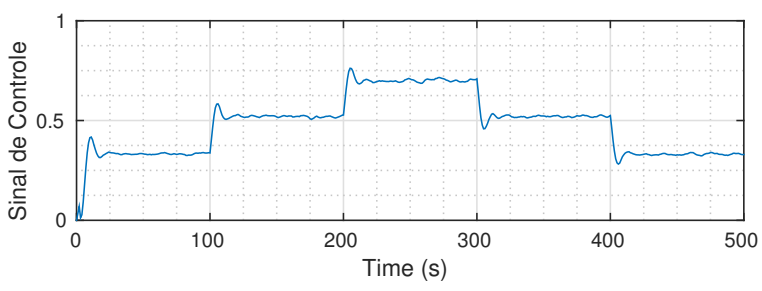

(a) Referência Escada

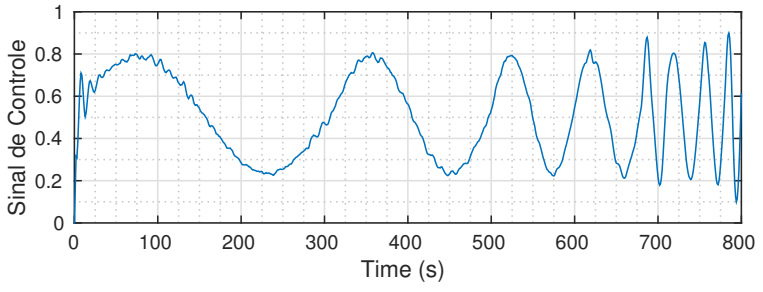

(b) Referência Senoidal

Figura 2. Rastreamento do controlador MFAC com distúrbios no sinal de saída, para os sinais de referência escada e senoidal, e sinais de controle.

Tabela 1. Características de resposta para diferentes soluções considerando diferentes escolhas do conjunto de Pareto aproximado.

\begin{tabular}{|c|c|c|c|c|c|c|}
\hline & \multicolumn{6}{|c|}{ Sem ruído } \\
\hline & \multicolumn{3}{|c|}{ Escada } & \multicolumn{3}{|c|}{ Senoidal } \\
\hline & $S_{S o l}^{T D}$ & $\mathrm{Sol}_{f_{1}}$ & $\mathrm{Sol}_{f_{2}}$ & $S_{S o l}^{T D}$ & $\mathrm{Sol}_{f_{1}}$ & $\mathrm{Sol}_{f_{2}}$ \\
\hline$f_{1}$ & 0,00034 & 0,00024 & 0,00093 & 0,00016 & 0,00015 & 0,00018 \\
\hline$f_{2}$ & 0,00015 & 0,00016 & 0,00011 & 0,00008 & 0,00010 & 0,00003 \\
\hline $\max |e|$ & 0,13056 & 0,11600 & 0,14583 & 0,15330 & 0,15330 & 0,15330 \\
\hline \multirow[t]{4}{*}{$\underline{t(\max |e|)}$} & 6 & 5 & 6 & 1 & 1 & 1 \\
\hline & \multicolumn{6}{|c|}{ Com ruído } \\
\hline & & Escada & & & Senoidal & \\
\hline & $S_{o l} l_{T D}$ & $\operatorname{Sol}_{f_{1}}$ & $\mathrm{Sol}_{f_{2}}$ & $\operatorname{Sol}_{T D}$ & $\operatorname{Sol}_{f_{1}}$ & $\mathrm{Sol}_{f_{2}}$ \\
\hline$f_{1}$ & 0,00085 & 0,00062 & 0,06495 & 0,00100 & 0,00066 & 0,08104 \\
\hline$f_{2}$ & 0,00040 & 0,00055 & 0,00026 & 0,00037 & 0,00054 & 0,00028 \\
\hline $\max |e|$ & 0,20004 & 0,13259 & 0,36460 & 0,28500 & 0,15330 & 0,41460 \\
\hline$t(\max |e|)$ & 202 & 6 & 202 & 78 & 1 & 526 \\
\hline
\end{tabular}

\subsection{Comparação com Controladores da Literatura}

O desempenho de rastreamento do controlador MFAC com o ajuste dos parâmetros usando GPR-EA, foi compa-
Tabela 2. Melhor solução escolhida pelo tomador de decisão.

\begin{tabular}{c|cc|cc}
\hline & \multicolumn{2}{|c|}{ Sem ruído } & \multicolumn{2}{c}{ Com ruído } \\
& Ref. Escada & Ref. Senoidal & Ref. Escada & Ref. Senoidal \\
\hline$\rho$ & 0,98 & 1,00 & 0,65 & 1,00 \\
$\eta$ & 1,97 & 1,9302 & 2 & $2,22 \times 10^{-16}$ \\
$\mu$ & $8,64 \times 10^{-3}$ & 0,4587 & 5,65 & 10 \\
$\lambda$ & 0,5370 & $2,22 \times 10^{-16}$ & $2,22 \times 10^{-16}$ & $2,22 \times 10^{-16}$ \\
\hline
\end{tabular}

rado com model based SVR controller (MB-SVR) (Uçak and Günel, 2016), SVM-based PID controller (SVM-based PID) (Iplikci, 2010), e Model free SVR controller (MFSVR) (Uçak and Günel, 2019). As comparações envolvem os rastreamento dos algoritmos de controle livre de modelo e baseado em modelo.

A média e variância da diferença entre o sinai de referência e o sinal de saída, e a média e variância do sinal de controle para todos os controladores são comparados (Tabela 3). Estas comparações incluem casos com e sem ruído. Todos 
Tabela 3. Média e variância da diferença entre o sinal de referência e o sinal de saída, e média e variância do sinal de controle para os controladores comparados, com sinais de referência escada e senoidal com e sem ruído.

\begin{tabular}{|c|c|c|c|c|c|c|c|c|}
\hline & \multicolumn{4}{|c|}{ Sem ruído } & \multicolumn{4}{|c|}{ Com ruído } \\
\hline & \multicolumn{2}{|c|}{ Ref. Escada } & \multicolumn{2}{|c|}{ Ref. Senoidal } & \multicolumn{2}{|c|}{ Ref. Escada } & \multicolumn{2}{|c|}{ Ref. Senoidal } \\
\hline & $\mu$ & $\sigma^{2}$ & $\mu$ & $\sigma^{2}$ & $\mu$ & $\sigma^{2}$ & $\mu$ & $\sigma^{2}$ \\
\hline \multicolumn{9}{|c|}{ Diferença Entre o Sinai de Referência e o Sinal de Saída } \\
\hline MB-SVR & 0,0034 & $1,0760 \times 10^{-4}$ & 0,0015 & $1,4026 \times 10^{-4}$ & $-0,0021$ & $1,8360 \times 10^{-4}$ & 0,0024 & $1,6055 \times 10^{-4}$ \\
\hline SVM-based PID & $-0,0013$ & $3,2003 \times 10^{-4}$ & $-1 \times 10^{-4}$ & $4,3985 \times 10^{-4}$ & $-0,0016$ & $3,2931 \times 10^{-4}$ & $-0,0010$ & $4,9318 \times 10^{-4}$ \\
\hline MF-SVR & 0,0079 & 0,0002 & 0,0019 & 0,0002 & 0,0080 & 0,0002 & 0,0027 & 0,0002 \\
\hline MFAC-(GPR-EA) & 0,0008 & 0.0002 & 0,0006 & 0.0001 & 0,0009 & 0.0003 & 0,0007 & 0.0002 \\
\hline \multicolumn{9}{|c|}{ Sinal de Controle } \\
\hline MB-SVR & 0,4730 & 0,0185 & 0,5354 & 0,0379 & 0,4828 & 0,0240 & 0,5338 & 0,0373 \\
\hline SVM-based PID & 0,4829 & 0,0177 & 0,5385 & 0,0368 & 0,4835 & 0,0178 & 0,5400 & 0,0377 \\
\hline MF-SVR & 0,4656 & 0,0200 & 0,5349 & 0,0379 & 0,4654 & 0,0204 & 0,5334 & 0,0374 \\
\hline MFAC-(GPR-EA) & 0,4772 & 0,0135 & 0,5134 & 0,0217 & 0,4774 & 0,0146 & 0,5131 & 0,0204 \\
\hline
\end{tabular}

os controladores apresentam desempenho aceitável, tendo o MFAC o melhor desempenho com variância menor com respeito aos outros controladores, incluído aquele baseado em modelo (MB-SVR). Geralmente, este tipo de controlador apresenta resultados de rastreamento melhores do que o MFAC, já que o comportamento dinâmico do sistema é utilizado no algoritmo. No entanto, os resultados mostrados na Tabela 3 sugerem que os parâmetros encontrados com o GPR-EA, permitem que o MFAC atinja um desempenho melhor do que os seus competidores. Em relação ao sinal de controle, observamos valores similares na média em todos os controladores. No entanto, o MFAC possui a menor variância em todos os casos testados.

\section{CONCLUSÕES}

Este artigo tratou do problema do controle adaptativo livre de modelo com e sem perturbação para um Reator Tanque Agitado Contínuo. O GPR-EA, que inclui o conhecimento de gerações passadas, é usado para ajustar os parâmetros de um MFAC. Nos experimentos pode-se observar a eficácia do controle MFAC no rastreamento de diferentes sinais de referência, com o ajuste realizado usando GPR-EA. As simulações ilustram um bom desempenho do controle para o caso de estudo na presença de distúrbios no processo, situação que é muito frequente nos sistemas reais. Na comparação do MFAC com algoritmos de controle livre de modelo e baseado em modelo, sugere uma melhoria no desempenho do MFAC ajustando os parâmetros deste controlador com GPR-EA.

\section{AGRADECIMENTOS}

Este trabalho foi apoiado por uma bolsa de estudos da CAPES.

\section{REFERÊNCIAS}

Chen, C., Xueyuan, L., Ye, Y., Jiarong, X., Zuwei, L., Xinggao, L., Jinshui, C., and Jiangang, L. (2019). Parameter self-tuning of siso compact-form model-free adaptive controller based on neural network with system error set as input. In 2019 12th Asian Control Conference $(A S C C), 468-473$. IEEE.

de Sousa, J.T.G., da Franca, J.E.M., and Araújo, A.F.R. (2015). Differential evolution-based parameter tuning in model-free adaptive control. In 2015 IEEE International Conference on Systems, Man, and Cybernetics, 17261731. IEEE. dos Santos Coelho, L. and Coelho, A.A.R. (2009). Modelfree adaptive control optimization using a chaotic particle swarm approach. Chaos, Solitons \& Fractals, 41(4), 2001-2009.

Guerrero-Peña, E. and Araújo, A.F.R. (2018). Multiobjective evolutionary algorithm with gaussian process regression. In 2018 IEEE Congress on Evolutionary Computation (CEC), 1-8. IEEE.

Hou, Z. and Jin, S. (2013). Model Free Adaptive Control: Theory and Applications. CRC Press.

Iplikci, S. (2010). A comparative study on a novel modelbased pid tuning and control mechanism for nonlinear systems. International Journal of Robust and Nonlinear Control, 20(13), 1483-1501.

Ji, C., Wang, J., Cao, L., and Jin, Q. (2014). Parameters tuning of model free adaptive control based on minimum entropy. IEEE/CAA Journal of Automatica Sinica, $1(4), 361-371$.

Liang, J., Zhu, X., Yue, C., Li, Z., and Qu, B. (2018). Performance analysis on knee point selection methods for multi-objective sparse optimization problems. In 2018 IEEE Congress on Evolutionary Computation (CEC), 1-8. IEEE.

Lloyd, S. (1982). Least squares quantization in pcm. IEEE Transactions on Information Theory, 28(2), 129-137.

Rasmussen, C.E. and Williams, C.K. (2006). Gaussian Processes for Machine Learning, volume 1. MIT Press Cambridge.

Roman, R.C., Radac, M.B., Precup, R.E., and Petriu, E.M. (2016). Data-driven model-free adaptive control tuned by virtual reference feedback tuning. Acta Polytechnica Hungarica, 13(1), 83-96.

Uçak, K. and Günel, G.Ö. (2016). An adaptive support vector regressor controller for nonlinear systems. Soft Computing, 20(7), 2531-2556.

Uçak, K. and Günel, G.Ö. (2019). Model free adaptive support vector regressor controller for nonlinear systems. Engineering Applications of Artificial Intelligence, 81, $47-67$.

Wang, J., Ji, C., Cao, L., and Jin, Q. (2012). Model free adaptive control and parameter tuning based on second order universal model. Journal of Central South University (Science and Technology), 43(5), 1795-1802.

While, L., Hingston, P., Barone, L., and Huband, S. (2006). A faster algorithm for calculating hypervolume. IEEE Transactions on Evolutionary Computation, 10(1), 2938 . 